\title{
A Review of Research on Traffic Modeling for NoC
}

\author{
Haiyun $\mathrm{Gu}^{\mathrm{a}}$, Hua Bo ${ }^{\mathrm{b}}$, Lei Ren \\ Department of Electrical Engineering, Shanghai Maritime University, Shanghai, 210306, China \\ aemail: hygu@shmtu.edu.cn, bemail: huabo@shmtu.edu.cn
}

Keywords: Network-on-Chip; Traffic modeling; Application specific

\begin{abstract}
NoC (Network on Chip), as a promising interconnection infrastructure for MPSoC (Multi-Processor System-on-Chip), has been the research focus since its invention. Accurate traffic models are necessary to deplore the vast design space of NoC. In this paper, we survey the researching works on the traffic modeling of NoC by referring and comparing to the development pattern of the traffic models in packet-switched computer networks, and try to figure out the direction of the next step.
\end{abstract}

\section{Introduction}

With the significantly increasing amount of hardware resources on a single chip, the greatest challenge for MPSoC designers shifts from computation to communication. NoC is a promising approach to provide a high performance interconnection architecture for MPSoC [1].

From its invention around the year 2000, NoC borrowed many concepts and techniques from the well-established domain of packet-switched computer networks. A lot of researches and design efforts in the area of computer networks have demonstrated that an efficient traffic model, which can correctly describe the traffic behavior, helps designers to determine the optimal architecture and evaluate the performance [2]. In the same way, efficient traffic models should be essential to design and evaluate the performance of an on-chip network.

This paper presents a review of current research on NoC traffic modeling. Section 2 introduces the development pattern of the traffic modeling for computer networks as a reference. Section 3 analyzes the current research approaches in traffic modeling for NoC, and discusses the trends and open issues in this field. Finally we conclude the paper.

\section{Traffic Modeling for Computer Networks}

The concept of NoC was originated from the computer networks. So it is natural to refer to the traffic models developed in the traditional telecommunication and computer networks. The aim of traffic modeling is to find stochastic processes to represent the behavior of traffic [3]. Through decades of development, technologies of traffic modeling were originated from the circuit-switched telecommunication networks, adapted to the packet-switched computer networks, and being applied to the network-on-chip.

\section{Traditional possion traffic models:}

From the 1910s, A.K.Erlang famously applied the poisson distribution to the random telephone traffic in the circuit-switched networks. Over several decades, Erlang's work stimulated the use of queuing theory.

\section{Self-similar traffic models:}

Since the 1970s, packet-switched networks started to be popular. Traditional poisson traffic model couldn't be applied directly to the packet-switched networks. Leland et al. showed that traffic process $X_{n}$ for packet networks was a LRD(Long Range Dependent) processes [4].

Essentially, self-similarity means that the rescaled process $a^{-\mathrm{H}} \mathrm{X}_{\text {an }}$ has the same statistical properties as the original process $\mathrm{X}_{\mathrm{n}}$ over a wide range of scales a $>0$ and some parameter $0<\mathrm{H}<1$. The Hurst parameter $\mathrm{H}$ is a measure of self-similarity. For $0.5<\mathrm{H}<1$, the traffic process is a LRD process. Self-similarity and LRD are important statistical characteristics because they believed to 
have a major effect on queueing performance. Bursts of LRD traffic tends to be more persistent, thereby causing longer queue lengths than estimated for traditional SRD traffic such as poisson.

Since then, by measuring and analyzing the traffic traces of various networks, significant advances have been made in LRD, wavelet, and multifractal traffic modeling approaches. Multifractal models have local scaling properties dependent on a timevarying $h(t)$, the local Hölder exponent, instead of a constant Hurst parameter. Intervals where $\mathrm{h}(\mathrm{t})<1$ show bursts while intervals where $h(t)>1$ correspond to small fluctuations. In traffic measurements of internet, the local scaling $\mathrm{h}(\mathrm{t})$ appears to change randomly in time.

\section{Application-specific traffic models:}

Traffic analysts have attempted to build specific traffic models for most popular applications (web, ftp, email, video, p2p, etc.). In the recent decade, there are many new multimedia applications (instant message, p2p file distribution, VoIP, online games, etc.). [5] showed the coexistence of poissonity and self-similarity in the traffic of web and p2p.

From the above introductions, we can summarize that the development procedure of traffic modeling for computer networks includes 3 stages:

$\checkmark \quad$ Using existing traffic models: possion model;

$\checkmark$ Characterizing the real traffic traces: LRD, wavelet, and multifractal traffic models;

$\checkmark$ Developing more specific traffic models to describe different applications.

Apparently, traffic modeling is an on-going research because of the constant evolution of networks and applications.

\section{Traffic Modeling for NoC}

Many concepts in NoC are originated in the packet-switched computer networks. So it is logical that the research on the traffic modeling for NoC may have the similar development pattern.

\section{Using existing traffic models:}

In the early NoC studies, researchers used the common traffic models in computer network simulators to evaluate NoC performance, including: uniform, possion, exponential, on/off, normal, CBR, etc. Table 1 lists some of the NoC simulators and their traffic models. However, a realistic traffic model of NoC is necessary and essential for the accurate evaluation.

Table 1. List of some traffic models used in NoC simulators

\begin{tabular}{|c|c|c|c|c|}
\hline NoC simulators & Language & topology & $\begin{array}{c}\text { Routing, } \\
\text { switching }\end{array}$ & Traffic model \\
\hline Booksim[6] & C++ & $\begin{array}{c}\text { 2D mesh, } \\
\text { torus, trees, etc. }\end{array}$ & $\begin{array}{c}\text { dimensional } \\
\text { order, VC, etc. }\end{array}$ & uniform, etc. \\
\hline OPNEC-Sim[7] & C++ & $\begin{array}{c}\text { 2D, 3D mesh, } \\
\text { torus, ring, } \\
\text { shared-bus }\end{array}$ & $\begin{array}{c}\text { XY, adaptive } \\
\text { Odd-Even }\end{array}$ & uniform \\
\hline OMNET++[8] & C++ & 2D torus & wormhole & exponential \\
\hline Nostrum[9] & $\begin{array}{c}\text { SystemC } \\
\text { and Python }\end{array}$ & $\begin{array}{c}\text { Mesh, torus, } \\
\text { tree, ring }\end{array}$ & XY,VC & constant rate \\
\hline Noxim[10] & SystemC & mesh & XY, adaptive & $\begin{array}{c}\text { possion, } \\
\text { on/off }\end{array}$ \\
\hline Nirgam[11] & SystemC & Mesh, torus & $\begin{array}{c}\text { Source, XY, } \\
\text { constant rate, burst, } \\
\text { input trace } \\
\text { adaptive, } \\
\text { wormhole }\end{array}$ & $\begin{array}{c}\text { XY, VC } \\
\text { uniform random, } \\
\text { tornado }\end{array}$ \\
\hline GARNET[12] & C++ & any & \multicolumn{2}{|c|}{} \\
\hline
\end{tabular}

\section{Real application traffic trace pattern of NoC:}

Varatkar et al [13] demonstrated the traffic between different modules for an MPEG-2 decoder on the NoC platform exhibited long-range dependence, and presented a synthetic traffic generator structured on the Hurst parameter $\mathrm{H}$. 
Since then, the research on traffic modeling of NoC focused on the analysis of real application traffic trace. Soteriou et al [14] proposed a 3-tuple statistical traffic model $(\mathrm{H}, \mathrm{p}, \sigma)$ and captured the self-similarity of the traffic set derived from 30 actual traffic traces gathered from full system simulations. Bahn et al [15] also presented a self-similar generic traffic model (Hs, $\lambda s, \delta(s, d)$ ). Liu et al [16] presented an available NoC traffic suite MCSL based on 8 real applications implemented on 3 topologies of NoC platform. Table 2 lists some details.

Table 2. Real application traffic trace pattern of NoC

\begin{tabular}{|c|c|c|c|}
\hline Researchers & Traffic model & Apps source & Topology \\
\hline $\begin{array}{l}\text { Soteriou V, Wang H, } \\
\text { Peh L S. } \\
{[14]}\end{array}$ & $\begin{array}{l}(\mathrm{H}, \mathrm{p}, \sigma) \\
\mathrm{H}: \text { temporal burstiness } \\
\mathrm{p}: \text { spatial hop destribution } \\
\sigma: \text { spatial injection distribution }\end{array}$ & $\begin{array}{c}30 \text { traffic traces } \\
\text { from } \\
\text { TRIPS CMP, } \\
\text { RAW CMP, } \\
\text { DCC CMP. }\end{array}$ & $\begin{array}{l}5 * 5 \text { mesh; } \\
4 * 4 \text { mesh }\end{array}$ \\
\hline $\begin{array}{l}\text { Bahn J H, } \\
\text { Bagherzadeh N. } \\
\text { [15] }\end{array}$ & $\begin{array}{l}(\mathrm{Hs}, \lambda \mathrm{s}, \delta(\mathrm{s}, \mathrm{d})) \\
\text { Hs: temporal burstiness } \\
\lambda \mathrm{s} \text { : injection rate } \\
\delta(\mathrm{s}, \mathrm{d}) \text { : spatial distribution }\end{array}$ & $\begin{array}{l}8 \text { traffic traces } \\
\text { from } \\
\text { SPLASH-2 }\end{array}$ & $7 * 7$ mesh \\
\hline $\begin{array}{l}\text { Liu W, Xu J, Wu X, } \\
\text { et al. } \\
{[16]}\end{array}$ & $\begin{array}{l}\text { STP(Statistical traffic pattern) } \\
\text { RTP(Recorded traffic pattern) }\end{array}$ & $\begin{array}{l}8 \text { traffic models } \\
\text { from } \\
\text { real applications }\end{array}$ & $\begin{array}{l}4 * 4,4 * 8 \text {, and } \\
8 * 8 \text { for mesh and } \\
\text { torus; } 16,32 \text {, and } \\
64 \text { PBs fattree. }\end{array}$ \\
\hline
\end{tabular}

Additionally, just like the situation in the computer networks, there were researches on multifractal approach to traffic modeling for NoC. Bogdan [17] proposed a statistical physics inspired approach to capture the non-stationary effects in NoC traffic. He also presented a novel quantum-leap inspired model to account for non-stationarity observed in packet arrival processes [18].

\section{Application-specific traffic models for NoC}

The self-similarity of the traffic models in the computer networks may be subjected to access control, flow control, congestion control, and contention within the network from other packet flows [3]. A natural question is what parameters can impact on the self-similarity of the NoC traffic models. [19-20] suggested that the specific application had a significant relationship with the Hurst parameter. [21] demonstrated that increasing channel service rate of NoC reduced self-similarity degree. Although the development procedure of the traffic modeling in NoC is similar to it in the computer networks, it is impractical to blindly reuse features of application-specific traffic models in computer networks. So far, most research on the real application traffic trace of NoC has been based on specific applications which make it difficult to compare or reproduce. Benchmarks of NoC are necessary.

\section{Conclusion}

In this paper, we have summarized the studies on traffic modeling for NoC. By referring and comparing to the development pattern of traffic modeling for computer networks, we have discussed the current state of researches on real application traffic traces, and presented the need of application-specific traffic benchmarks for NoC.

At last, we want to mention a theory named Network Calculus which has been developed to deal with the queueing type problems in packet switched networks. [22-24] applied this theory into NoC traffic model to analysis the worst-case performance bounds. Further studies are needed to tailor the theory results to analyze the real traffic in NoC. Combining the theory limits and simulation results based on the application-specific traffic models can be very helpful to direct the NoC design. 


\section{Acknowledgement}

In this paper, the research was sponsored by Science \& Technology Program of Shanghai Maritime University (Project No. 20120066).

\section{References}

[1] Dally W J, Towles B. Route packets, not wires: on-chip inteconnection networks[C]// IEEE, 2001:684-689.

[2] Barakat C, Thiran P, Iannaccone G, et al. Modeling Internet backbone traffic at the flow level[J]. IEEE Transactions on Signal Processing, 2003, 51(8):2003.

[3] Thomas M. Chen, Network Traffic Modeling, Chapter in the Handbook of Computer Networks, Hossein Bidgoli (ed.), Wiley, 2007.

[4] Tranter W H, Taylor D P, Ziemer R E, et al. On the SelfSimilar Nature of Ethernet Traffic (Extended Version)[C]// Wiley-IEEE Press, 2007.

[5] Gupta H, Mahanti A, Ribeiro V J. Revisiting coexistence of poissonity and self-similarity in Internet traffic [J]. 2009, 33(1):1-10.

[6] Jiang N, Balfour J, Becker D U, et al. A detailed and flexible cycle-accurate Network-on-Chip simulator[C]// IEEE International Symposium on PERFORMANCE Analysis of Systems and Software. 2013:86 - 96.

[7] Cai J, Huang G, Wang S, et al. OPNEC-Sim: An efficient simulation tool for Network-on-Chip communication and energy performance analysis[C]// IEEE International Conference on Solid-State and Integrated Circuit Technology. IEEE, 2010:1892 - 1894.

[8] Al-Badi R, Al-Riyami M, Alzeidi N. A parameterized NoC simulator using OMNet++[C]// International Conference on Ultra Modern Telecommunications \& Workshops. 2009:1-7.

[9] Lu Z, Thid R, Millberg M, et al. NNSE: Nostrum network-on-chip simulation environment[J]. Proc of Ssocc, 2012.

[10] http://noxim.sourceforge.net/

[11] http://nirgam.ecs.soton.ac.uk/

[12] Kumar A, Agarwal N, Peh L S, et al. A system-level perspective for efficient NoC design[J]. 2008:1-5.

[13] Varatkar G V, Marculescu R. On-chip traffic modeling and synthesis for MPEG-2 video applications[J]. IEEE Transactions on Very Large Scale Integration Systems, 2004, 12(1):108-119.

[14] Soteriou V, Wang H, Peh L S. A Statistical Traffic Model for On-Chip Interconnection Networks[J]. 2006:104-116.

[15] Bahn J H, Bagherzadeh N.. A Generic Traffic Model for On-Chip Interconnection Networks[J]. in The First International Workshop on Network-on-Chip Architectures, 2011.

[16] Liu W, Xu J, Wu X, et al. A NoC Traffic Suite Based on Real Applications[J]. 2011, 8(1):66-71.

[17] Bogdan P, Marculescu R. Non-Stationary Traffic Analysis and Its Implications on Multicore Platform Design[J]. IEEE Transactions on Computer-Aided Design of Integrated Circuits and Systems, 2011, 30(4):508-519.

[18] Bogdan P, Kas M, Marculescu R, et al. QuaLe: A Quantum-Leap Inspired Model for 
Non-stationary Analysis of NoC Traffic in Chip Multi-processors[C]// ACM/IEEE International Symposium on Networks-On-Chip. IEEE Computer Society, 2010:241-248.

[19] Chen Y, Hu J, Wang J. Self-similar traffic study of on-chip interconnection networks[M]. 2013.

[20] Qin M W, Hu J H, Ma S. Affect analysis of FFT algorithm length on traffic self-similarity in NOC[C]// International Computer Conference on Wavelet Active Media Technology and Information Processing. IEEE, 2013:149-152.

[21] Khonsari A, Aghajani M R, Tavakkol A, et al. Mathematical analysis of buffer sizing for Network-on-Chips under multimedia traffic[J]. 2008:150-155.

[22] Bakhouya M, Suboh S, Gaber J, et al. Analytical modeling and evaluation of On-Chip Interconnects using Network Calculus[C]// ACM/IEEE International Symposium on Networks-On-Chip. IEEE Computer Society, 2009:74-79.

[23] Qian Y, Lu Z, Dou W. Analysis of worst-case delay bounds for on-chip packet-switching networks[J]. IEEE Transactions on Computer-Aided Design of Integrated Circuits and Systems, 2010, 29(5):802-815.

[24] Chen J W, Tang L, Xi H S, et al. A stochastic network calculus based approach for on-chip networks[J]. 2011:4545-4549. 